\title{
Frecuencia de alteraciones citológicas cervicales y virus papiloma humano en una muestra de estudiantes universitarias en Temuco, Chile
}

\author{
Angélica Melo ${ }^{1-6}$, Sonia Montenegro², Sandra Liempi ${ }^{3}$ Sergio Moreno ${ }^{3}$, Tiare de La Barra², \\ Pablo Guzmán ${ }^{1-3}$, Luis Bustos ${ }^{4}$ y Flery Fonseca-Salamanca ${ }^{5-6}$
}

\section{Frequency of cervical cytological alterations and human papilloma virus in a sample of university students in Temuco, Chile}

Background: The early onset of sexual activity can promote the development of cervical alterations and sexually transmitted infections, especially the human papillomavirus (HPV) very common in adolescents and young people. Aim: The condition of the cervix, HPV and sexual behavior in young women under 25 years of age were analyzed. Methods: 182 university students, healthy, sexually active, 18-24 years old, without vaccine for HPV participated. Papanicolaou (Pap) test and classification of high and low risk HPV (HR and LR) were performed by real time polymerase chain reaction. The sexual behaviors were consulted in private. Results: The $46.9 \%$ of Pap presented cytological alterations, non-specific inflammation/hemorrhagic (29.4\%) and atypical smear (10.2\%) being de most frequent. The overall frequency of HPV-HR was $24.3 \%$, of these $67.4 \%$ presented an altered Pap. There was an association between cytological alterations and HPV $(\mathrm{p}<0.0001)$ and years of sexual activity and atypical smear or cervical intraepithelial neoplasia grade I (CIN I) $(\mathrm{p}=0.009) .11 .9 \%$ of young women $(21 / 177)$ presented atypical smear or CIN I, with $66.7 \%$ of cases HPV-HR. Conclusions: These findings alert the vulnerability of these young women who would have a potential risk of viral persistence, CIN and eventually cancer. It is important to emphasize counseling and prevention prior to the regular age of admission to the screening program for cervical cancer. This study was financed by the Universidad de La Frontera through Projects DI15-0047 and DI17-0123.

Keywords: Papanicolaou; cervical cytology; human papilloma virus; young women; university students; sexual behavior.

Palabras clave: Papanicolaou; citología cervical; virus papiloma humano; mujeres jóvenes; estudiante universitaria; conductas sexuales.

\section{Introducción}

$\mathrm{E}$ n Chile, el programa de tamizaje del cáncer cervico-uterino(CCU) recomienda realizar el examen citológico de Papanicolaou (Pap), que analiza la morfología celular exo-endocervical, a partir de los 25 años ${ }^{1}$. Sin embargo, en la adolescencia durante el proceso fisiológico de maduración de la zona de transformación del cuello uterino, se puede favorecer el desarrollo de alteraciones cervicales e infecciones de transmisión sexual (ITS) ${ }^{2}$. En particular, la infección por el virus papiloma humano (VPH) es una de las ITS más frecuente en adolescentes y jóvenes ${ }^{3}$.

Por otro lado, como ya es conocido, la presencia de genotipos de alto riesgo oncogénico de VPH (VPH-AR) en la mucosa cervical, están fuertemente implicados en la etio-patogenia del CCU y sus lesiones precursoras ${ }^{4}$. No obstante, para alcanzar la etapa de carcinoma, es necesario la presencia de factores de riesgo como: edad de inicio de actividad sexual, tiempo de persistencia del VPH en la mucosa cervical, múltiples parejas sexuales, co-infección con otros microorganismos, entre otros ${ }^{5-8}$.

En la Región de La Araucanía se han publicado estudios sobre el VPH y en mujeres menores de 25 años pesquisadas en la Unidad de Patología Cervical'; sin embargo, no hay estudios publicados en mujeres jóvenes sexualmente activas y sanas. Por lo tanto, el propósito fue determinar el estado del cuello uterino por el Pap y la presencia del VPH asociada a conductas sexuales en una muestra de mujeres universitarias de Temuco menores de 25 años.

\section{Materialles y Métodos}

\section{Pacientes}

Participaron voluntariamente 182 mujeres sanas, sexualmente activas, menores de 25 años y no vacunadas
Departamento Anatomía Patológica.

${ }^{2}$ Departamento de Especialidades. Laboratorio de Diagnóstico Clínico Molecular. Facultad de Medicina. Universidad de Concepción. Concepción. Chile 3Unidad de Anatomía PatológicaCitología del Hospital Hernán Henríquez Aravena Temuco. Chile.

${ }^{4}$ Departamento de Salud Pública y CIGES.

${ }^{5}$ Departamento de Ciencias Preclínicas.

${ }^{6}$ Laboratorio de

Inmunoparasitología Molecular, Centro de Excelencia en Medicina Traslacional, Núcleo Científico Tecnológico en Biorecursos (CEMT-BIOREN). Facultad de Medicina. Universidad de La Frontera, Temuco, Chile.

Esta investigación fue financiada por la Universidad de La Frontera a través de los Proyectos DI150047 y DI17-0123.

Recibido: 24 de julio de 2018 Aceptado: 24 de marzo de 2019

Correspondencia a: Angélica Melo Angermeyer angelica.melo@ufrontera.cl 
para el VPH. En el período 2013-2015 se distribuyeron afiches en universidades de Temuco (Universidad Mayor, U. Santo Tomás, U. Católica, U. Autónoma, U. de La Frontera), invitando a jóvenes a realizarse un Pap sin costo y a participar en este estudio. Previo al enrolamiento y obtención de la muestra cervical, se obtuvo un consentimiento informado aprobado por el Comité de Ética Científica de la Universidad de La Frontera.

\section{Conductas sexuales}

Cada participante respondió privadamente un cuestionario sobre edad de inicio de actividad sexual, número de parejas sexuales (últimos 3 años), métodos de prevención de embarazo y de ITS y antecedentes de Pap previos.

\section{Muestras de Pap}

Las muestras cervicales fueron colectadas por una matrona mediante un cepillado (citobrush) de la zona exoendocervical. De inmediato, se realizó el extendido sobre un portaobjeto cubriendo la muestra con un citofijador, se dejó secar y se aplicó la tinción de Pap convencional ${ }^{10}$. El citobrush se colocó en $3 \mathrm{ml}$ de tampón fosfato salino (PBS1X) para su traslado al laboratorio. Se dividieron los $3 \mathrm{ml}$ en dos tubos de $1,5 \mathrm{ml}$ y por centrifugación se obtuvo un concentrado celular que fue tratado con solución de lisis (kit E.Z.N.A. ${ }^{\circledR}$ Tissue DNA) y almacenado a $-80^{\circ} \mathrm{C}$ hasta su uso.

\section{Extracción del ADN}

Se realizó con el kit E.Z.N.A. ® Tissue DNA (Omega Bio- Tek USA), según instrucciones del fabricante. Sólo se utilizaron muestras con ADN amplificable determinado por una reacción de polimerasa en cadena (RPC) simple dirigida a secuencias del gen $\beta$-globina, empleando los partidores PCO4 5'-caacttcatccacgttcacc-3' y GH20 5'-gaagagccaaggacaggtac-3', que amplifican un fragmento de 268 pares de bases (pb).

\section{Detección del VPH}

Se realizó mediante dos tipos de RPC, una de tamizaje mediante una RPC anidada convencional dirigida al gen L1 del VPH (RPC L1), utilizando los partidores externos MY11 y MY09 y los internos GP5+/GP6+, que amplifican un fragmento de $150 \mathrm{pb}^{11}$. Para la clasificación según riesgo oncogénico se utilizó una RPC anidada dirigida a secuencias de los genes E6/E7 que identifica los VPHAR-16, 18, 31, 33, 35, 52, 53, 56, 58, 59, 66 y los tipos virales de bajo riesgo (VPHBR) 6 y 11 . Para la primera amplificación (RPC convencional) se utilizaron los partidores externos: LCRS 5'-aagggagtaaccgaaaacggt-3 'y E7AS 5'-tcatcctcetcctctgag-3'. Con el producto RPC de la primera amplificación se realizó una segunda amplificación (RPC a tiempo real) utilizando una mezcla patrón comercial (HOT FIREP ${ }^{\circledR}$ EvaGreen $^{\circledR}$ qPCR mix plus (ROX) y los partidores internos: PU1M: 5'-tgtcaaaaaccgttgtgtcc-3 'y PU2R: 5' -gagctgtcgettaattgctc-3' para amplificar VPH-AR (Tubo AR) y los partidores PU31B: 5'-tgctaattcggtgctacctg-3' y PU2R: 5'-gagctgtcgettaattgctc-3 para los VPH-BR (Tubo BR) ${ }^{12-15}$. La sensibilidad de la RPC E6/E7 anidada combinando RPC convencional y en tiempo real fue coincidente con la reportada (una copia de VPH por célula) ${ }^{16}$. Se utilizaron como controles positivos plásmidos de genoma completo de VPH comerciales (ATCC) para VPH-BR 6 y 11 y VPH-AR 16 y 18.

\section{Lectura de los Pap}

Fue realizada por dos tecnólogos médicos (citotecnólogos). Cada uno leyó una serie de placas y los casos dudosos fueron definidos por el patólogo. El diagnóstico presuntivo de inflamación por Candida spp y de virus herpes simplex (VHS) se determinó al visualizar hifas o inclusiones intracelulares, respectivamente. Exclusión de casos: Se excluyeron frotis sin células endo-cervicales o metaplásicas, con fijación deficiente o muestra escasa, inadecuados para examen citológico.

Definiciones:

- Pap Normal: Ausencia de células neoplásicas o cualquier alteración citológica.

- Pap Alterado o alteraciones citológicas cervicales: Presencia de inflamación inespecífica/hemorrágico (Inf/Hem), inflamación presuntiva por Candida spp, signos de infección por VHS (Candida/VHS), signos morfológicos (coilocitos) de VPH (signos de VPH).

- Frotis Atípicos (FA, sin caracteres definidos o sin descartar lesión de alto grado cervical), neoplasia intraepitelial grado 1(NIE I).

Clasificación del Pap según lesión escamosa de bajo grado:

- $\operatorname{Pap}(+)$ : Pap positivo cuando las muestras presentaron FA o NIE I.

- Pap(-): Pap negativo cuando las muestras no presentaron FA o NIE I, aunque sí podían presentar otras alteraciones citológicas.

El procesamiento de las muestras (extracción del ADN y cribado de las muestras para el VPH) fue realizado en el Laboratorio de Inmunoparasitología Molecular del Centro de Excelencia en Medicina Translacional de la Universidad de La Frontera, la clasificación en VPH-AR y VPH-BR en el Laboratorio de Diagnóstico Clínico Molecular de la Universidad de Concepción y los Pap fueron realizados en la Unidad de Anatomía Patológica-Citología del Hospital Hernán Henríquez Aravena de Temuco.

Cada joven participante fue informada de los resultados de su muestra y se le entregó una consejería sobre lo que significaba el resultado del Pap y del VPH. 


\section{Análisis de resultados}

Todos los datos fueron codificados e ingresados en una base digital. Se realizó análisis descriptivo con tablas de contingencia de frecuencias absolutas y relativas. Los datos de las variables cuantitativas fueron obtenidos con los estadígrafos rango, promedio y desviación estándar (DE). Para la comparación de porcentajes se utilizó la prueba exacta de Fisher. Nivel de significación fue de $5 \%$.

\section{Resultados}

Participaron 182 mujeres jóvenes universitarias, asintomáticas, sin vacuna para VPH, de distintas carreras y universidades de la ciudad de Temuco. Sin embargo, el grupo de estudio quedó conformado por 177 jóvenes, debiéndose excluir cinco muestras que fueron inadecuadas para el examen de Pap. El rango de edad fue entre 18 y 24 años con un promedio de 21,5 $\pm 1,5$ años. La edad de inicio de actividad sexual fue entre 14 a 22 años, con un promedio de 17,5 $\pm 1,82$ años. El tiempo de actividad de sexual fue de 1 a 9 años, con un promedio de 4,0 \pm 1,9 años. Tenían tres o menos años de vida sexual activa $40,7 \%$ de las jóvenes.

\section{Diagnóstico de los Pap}

El 53,1\% (94/177) de las jóvenes presentó un Pap Normal y 46,9\% (83/177) alteraciones citológicas cervicales: 29,4\% inflamación inespecífica/hemorrágico, 2,3\% Candida/VHS, 3,4\% con signos de VPH, 10,2\% frotis atípico y $1,7 \%$ NIE I (Tabla 1 ). Al sumar los casos con frotis atípico más las NIE I, se obtuvo $11,9 \%$ de jóvenes con una lesión escamosa de bajo grado.
Todas las muestras que fueron positivas para la RPC L1 pudieron ser clasificadas en VPHAR y VPHBR mediante la RPC E6/E7 anidada a tiempo real.

Al comparar la clasificación de VPH entre Normal versus la alteración Candida/VHS y entre Normal versus frotis atípico, se encontraron diferencias significativas $(\mathrm{p}<0,05)$, siendo las otras comparaciones no significativas (Tabla 1). La frecuencia total de VPH fue $31,1 \%$ (55/177). Según el riesgo oncogénico, se observó para el total del grupo de estudio, $24,3 \%$ de casos con VPH-AR y $6,8 \%$ con VPH-BR. En las jóvenes con alteraciones citológicas o Pap alterado se observó 67,4\% (29/43) de VPH-AR y analizando el total de casos (VPHAR, VPHBR y negativos) en cada grupo con alteración citológica los porcentajes obtenidos para VPHAR fueron: inflamación inespecífica o hemorrágico 21,2\% (11/52), inflamación por Candida o VHS 75\% (3/4), signos de VPH $16,7 \%(1 / 6)$, frotis atípicos $66,7 \%(12 / 18)$ y NIE I $66,7 \%(2 / 3)$ (Tabla 1).

Se destaca el porcentaje de jóvenes con virus de AR $(66,7 \%, 14 / 21)$ entre las que tenían un frotis atípico o una NIE I, versus aquellas que no tenían un frotis atípico o una NIE I (18,6\%, 29/156) (Tabla 1).

La Tabla 2 muestra la asociación de las lesiones cervicales frotis atípico y NIE I [expresados como Pap $(+)$ ], VPH y conductas sexuales en las jóvenes universitarias. No se observó asociación significativa en las variables analizadas [Pap (+), Pap (-), VPH-AR, VPH-BR y conductas sexuales].

En la Tabla 3, se asocian años de actividad sexual, VPH y hallazgos al Pap. Se obtuvo una diferencia significativa $(p=0,009)$ en relación a jóvenes con un Pap $(+)$ que llevaban $\leq 3$ años de actividad sexual $(4,2 \%)$ versus aquellas con 4 a 9 años de actividad sexual (17,1\%).

\begin{tabular}{|c|c|c|c|c|c|}
\hline Alteraciones citológicas cervicales & Total & $\begin{array}{l}\text { Clasificación del VPH } \\
\text { AR }\end{array}$ & $\begin{array}{l}\text { N (\%) } \\
\text { BR }\end{array}$ & Neg & $\mathbf{P}^{*}$ \\
\hline Inflamación Inesp. o Hem & $52(29,4)$ & $11(21,2)$ & $2(3,8)$ & $39(75,0)$ & 0,432 \\
\hline Candida/VHS & $4(2,3)$ & $3(75,0)$ & & $1(25,0)$ & 0,028 \\
\hline NIE I & $3(1,7)$ & $2(66,7)$ & & $1(33,3)$ & 0,087 \\
\hline Total & $177(100)$ & $43(24,3)$ & $12(6,8)$ & $122(68,9)$ & \\
\hline
\end{tabular}

*Prueba Exacta de Fisher para asociar clasificación del VPH según alteraciones citológicas cervicales (normal vs cada alteración). Normal: frotis negativo para la presencia de células neoplásicas o algún tipo de alteración citológica. Inflamación inesp. o Hem: inflamación Inespecífica o hemorrágico (frotis con presencia de sangre). Candida/ VHS: frotis con Inflamación por Candida o virus herpes simplex. VPH: signos morfológicos (coilocitos) de infección por VPH. FA: frotis atípico, células escamosas atípicas de significado indeterminado o células escamosas atípicas, sin descartar una lesión escamosa de alto grado. NIE I: neoplasia intraepitelial grado I. VPH: virus papiloma humano. AR: VPH alto riesgo. BR: VPH bajo riesgo. Neg: muestra negativa a VPH. 


\begin{tabular}{|c|c|c|c|c|c|c|}
\hline Conducta sexual & $\begin{array}{c}\text { Total } \\
n=177 \\
n(\%)\end{array}$ & $\begin{array}{c}\text { Negativas a FA, } \\
\text { NIEI y VPH } \\
n=62 \\
n(\%)\end{array}$ & $\begin{array}{c}\text { Positivas a FA y } \\
\text { NIE I (Pap (+)) } \\
n=21 \\
n(\%)\end{array}$ & $\begin{array}{c}\text { Negativas a } \\
\text { VPHBR } \\
n=165 \\
n(\%)\end{array}$ & $\begin{array}{c}\operatorname{VPHAR}(+) \text { y } \\
\operatorname{Pap}(-) \\
n=29 \\
n(\%)\end{array}$ & $\begin{array}{c}\operatorname{VPHAR}(+) \text { y } \\
\operatorname{Pap}(+) \\
n=14 \\
n(\%)\end{array}$ \\
\hline \multicolumn{7}{|c|}{ No Parejas sexuales (últimos 3 años) } \\
\hline 1 & $68(38,4)$ & $21(30,9)$ & $8(11,8)$ & $63(92,7)$ & $8(11,8)$ & $6(8,8)$ \\
\hline 2 & $44(24,9)$ & $19(43,2)$ & $3(6,8)$ & $40(90,9)$ & $12(27,3)$ & $2(4,6)$ \\
\hline$\geq 3$ & $65(36,7)$ & $21(32,3)$ & $10(15,4)$ & $62(95,4)$ & $9(13,9)$ & $6(9,2)$ \\
\hline$p$ & & 0,395 & 0,443 & 0,603 & 0,092 & 0,686 \\
\hline \multicolumn{7}{|c|}{ Uso de métodos de prevención } \\
\hline Ninguno & $25(14,1)$ & $10(40,0)$ & $3(12,0)$ & $23(92,0)$ & $5(20,0)$ & $2(8,0)$ \\
\hline Preservativo & $27(15,3)$ & $9(33,3)$ & $2(7,4)$ & $26(96,3)$ & $6(22,2)$ & $1(3,7)$ \\
\hline Anticonceptivo & $102(57,6)$ & $37(36,3)$ & $14(13,7)$ & $94(92,2)$ & $16(15,7)$ & $9 \quad(8,8)$ \\
\hline Ambos & $23(13,0)$ & $5(21,7)$ & $2(8,7)$ & $22(95,7)$ & $2(8,7)$ & $2(8,7)$ \\
\hline $\mathrm{p}$ & & 0,559 & 0,85 & 0,904 & 0,575 & 0,918 \\
\hline \multicolumn{7}{|l|}{ Pap anteriores } \\
\hline 0 & $108(61,0)$ & $32(29,6)$ & $9(8,3)$ & $100(92,6)$ & $15(13,9)$ & $6(5,6)$ \\
\hline $1-2$ & $56(31,6)$ & $24(42,9)$ & $11(19,6)$ & $54(96,4)$ & $12(21,4)$ & $7(12,5)$ \\
\hline$\geq 3$ & $13(7,3)$ & $5(38,5)$ & $1 \quad(7,7)$ & $11(84,6)$ & $2(15,4)$ & $1 \quad(7,7)$ \\
\hline $\mathrm{p}$ & & 0,217 & 0,092 & 0,213 & 0,488 & 0,24 \\
\hline \multicolumn{7}{|c|}{$\begin{array}{l}\text { Datos expresados en frecuencias y porcentajes: } \mathrm{n}(\%) \text {. Prueba Exacta de Fisher. FA: Frotis atípico células escamosas atípicas de significado indeterminado o células escamosas } \\
\text { atípicas, sin descartar una lesión escamosa de alto grado. NIE I: neoplasia intraepitelial grado I. Pap (+): Pap positivo si las muestras presentaron FA o NIE I. Pap (-): Pap } \\
\text { negativo si las muestras no presentaron frotis atípicos o NIE I aunque si podían presentar otras alteraciones citológicas. Preservativo: condón masculino. Anticonceptivo: } \\
\text { incluye píldora, inyección, implante y DIU. Ambos: preservativo más algún anticonceptivo. Pap anteriores: número de Pap realizados por las participantes antes de entrar } \\
\text { en el estudio. }\end{array}$} \\
\hline
\end{tabular}

\begin{tabular}{|c|c|c|c|c|c|c|}
\hline \multicolumn{7}{|c|}{ Años de actividad sexual } \\
\hline & & $\leq 3$ & $>3$ & & & \\
\hline Alteración cervical & & n (\%) & n (\%) & p & $\mathbf{R P}$ & ICRP \\
\hline $\operatorname{Pap}(+)$ & $\begin{array}{l}\text { neg } \\
\text { pos }\end{array}$ & $\begin{array}{r}69 \\
3 \quad(95,8) \\
3(4,2)\end{array}$ & $\begin{array}{l}87(82,9) \\
18(17,1)\end{array}$ & 0,009 & 4,1 & $1,25-13,45$ \\
\hline VPH-AR (+)/Pap (+) & $\begin{array}{l}\text { no } \\
\text { sí }\end{array}$ & $\begin{array}{r}70(97,2) \\
2 \quad(2,8)\end{array}$ & $\begin{array}{l}93(88,6) \\
12(11,4)\end{array}$ & 0,046 & 4,1 & $0,94-17.8$ \\
\hline $\operatorname{VPH}-A R(+) / \operatorname{Pap}(-)$ & $\begin{array}{l}\text { no } \\
\text { sí }\end{array}$ & $\begin{array}{l}60(83,3) \\
12(16,7)\end{array}$ & $\begin{array}{l}88(83,8) \\
17(16,2)\end{array}$ & 1 & 0,97 & $0,49-1,90$ \\
\hline
\end{tabular}

Además, el porcentaje de jóvenes con Pap (+) y VPH-AR $(+)$ se asoció significativamente con años de actividad sexual $(p=0,046)$. Sin embargo, no se puede afirmar clínicamente que tener sobre tres años de actividad sexual sea un posible factor de riesgo de esta respuesta positiva

\section{(IC95\% RP: 0,94-17,8).}

En relación a conductas sexuales, destaca que $15,3 \%$ de las jóvenes usaba sólo preservativo, 57,6\% usaba sólo anticonceptivo y $38,9 \%$ se habían tomado el Pap entre una $\mathrm{y}$ tres veces previamente. 


\section{Discusión}

El inicio cada vez más precoz de actividad sexual en las adolescentes chilenas es un factor importante a considerar desde el punto de vista de salud pública en el ámbito de la prevención y promoción en relación a las ITS y CCU.

El presente estudio reporta resultados en un grupo de estudiantes universitarias menores de 25 años, sobre el estado de su cuello uterino, VPH y conductas sexuales.

En relación a los cinco casos que debieron excluirse por muestra inadecuada para lectura del Pap se realizaron los esfuerzos necesarios para repetirles la toma del Pap pero no hubo éxito.

Casi la mitad de las estudiantes universitarias (46,9\%) presentaron distintas alteraciones citológicas en su Pap con presencia de VPH-AR en $34,9 \%$ de estos casos. En adolescentes y jóvenes, ya se han reportado Pap con inflamaciones inespecíficas, presencia de alguna ITS, NIE o hasta un cáncer cervical ${ }^{9,17-19}$. Un estudio realizado en mujeres asintomáticas, reporta 59,6\% de Pap inflamatorios con cultivo positivo a microorganismos, siendo la vaginosis bacteriana el diagnóstico más frecuente ${ }^{20}$.

El 31,7\% de las estudiantes universitarias participantes en este estudio presentó Pap con inflamación inespecífica o hemorrágico o bien Candida sp o virus herpes simplex (con mayor número de positivos para VPH), ofreciendo este escenario, un mayor riesgo de infección con el VPH o co-infección con otra ITS. Además, estas alteraciones citológicas cervicales observadas en el Pap de una joven, podrían ser una señal que indicara que la joven está más expuesta o más susceptible a tener una infección, reinfección o auto-infección viral, debido a que estas alteraciones anteriormente mencionadas, pueden producir micro-erosiones en la mucosa cervical permitiendo al VPH alcanzar la capa basal de ésta. Un estudio reporta que $16,7 \%$ de las mujeres con inflamación persistente en el Pap tenían una $\mathrm{NIE}^{21}$. Por otro lado, se ha observado aumento de bacterias anaeróbicas en mujeres con VPH-AR y un aumento en la intensidad de una NIE asociada a mayor diversidad de la microbiota, sugiriendo que ésta, podría estar involucrada en la persistencia viral y la progresión de la lesión ${ }^{22-25}$.

En relación a la frecuencia total del VPH, ésta fue similar a lo descrito en otras investigaciones para esta población ${ }^{26-28}$. En la mayoría de las jóvenes con VPHAR se observaron desde Pap normales hasta NIE I. Sin embargo, los casos con frotis atipicos y NIE I presentaron una mayor proporción de casos con VPH-AR en relación a los casos con Pap Normal. Por otro lado, se observó diferencias significativas al comparar la clasificación del VPH entre un Pap Normal versus el grupo de jóvenes que tenían Candida/VHS y entre un Pap Normal versus un frotis atipico.

Castellsague y cols. ${ }^{29}$, observaron que más de $25 \%$ de las mujeres fue infectada con el VPH dentro del primer año de comenzada su actividad sexual y la infección se asoció fuertemente con el desarrollo de NIE. En ese sentido, las mujeres universitarias que tenían cuatro o más años de actividad sexual presentaron la probabilidad de tener un diagnóstico positivo a FA o NIE, 4,1 veces más que las jóvenes que tenían $\leq 3$ años, reafirmando que la variable años de actividad sexual sería un posible factor de riesgo para tener un Pap positivo a FA o NIE. Es por estos hallazgos, que este grupo de jóvenes conforma un grupo potencialmente en riesgo, si presentan persistencia o reinfección con otro tipo de VPH-AR.

En relación a conductas sexuales, VPH y Pap (Tabla 2) no se observó una asociación significativa entre las variables, solamente una tendencia $(p=0,09)$ en los grupos "Positivas a FA/NIE I y Pap anteriores" y "VPHAR (+)/Pap (-) y número de parejas sexuales". Por otra parte, más de un tercio de las jóvenes había tenido $\geq 3$ parejas sexuales en los últimos tres años, pero no por ello, se observaron más casos de VPH-AR $(+)$ con Pap (+) en relación al grupo que había tenido sólo una pareja. Asimismo, 61,0\% de las jóvenes se realizó el Pap por primera vez, a pesar que 58,2\% informaba ser sexualmente activa por más de tres años. Rodríguez y cols. $^{30}$, reportan que $75 \%$ de las mujeres no se realiza el Pap por falta de motivación, aun sabiendo la utilidad del examen. Por consiguiente, se podría especular que, en el grupo de estudiantes universitarias estudiado, un factor importante para realizarse un Pap sería la motivación, más que la falta de conocimiento, puesto que $85 \%$ de ellas sabia sobre las ITS (dato no publicado ni mostrado en los resultados) y casi $40 \%$ se había realizado un Pap a pesar que aún no tenían la edad para ingresar al programa de tamizaje del CCU normado en Chile. Y en relación a que sólo $28,8 \%$ tenía relaciones sexuales con preservativo (solo o junto al anticonceptivo),Villegas y cols. ${ }^{31}$, reportan que las jóvenes chilenas reconocen estar en riesgo de adquirir ITS/VIH. Sin embargo, alrededor de un tercio de las participantes manifestaron no sentirse preocupadas o poco preocupadas por adquirir una ITS o el VIH. Sobre esto, Eguiluz-Cárdenas y cols. ${ }^{32}$, reportan que el factor confianza y el conocer o no a la otra persona influye sobre el uso del preservativo.

Por otro lado, se puede mencionar como una limitante del presente estudio que la metodología utilizada no fue verificada para afirmar que amplifica los VPH-BR 26, 34, 43 y 70 y los VPH-AR 45, 51 y 68. Como así mismo, en la revisión realizada no se encontró alguna publicación que hubiera reportado la identificación de los tipos virales mencionados anteriormente mediante esta misma técnica.

Sin embargo, se puede comentar como una ventaja, que la metodología permite clasificar en alto y bajo riesgo en sólo dos amplificaciones, sin la necesidad de usar sondas para RLB (reverse line blot) o secuenciación o 
kit comerciales o enzimas de restricción (RFLP) que son métodos más complejos y de más alto $\operatorname{costo}^{33}$. Además, todos los casos amplificados por la RPC L1 fueron también amplificados por la RPC E6/E7 en tiempo real.

En conclusión, se identificó un grupo de jóvenes que presentó lesiones cervicales de bajo grado (FA y NIE I), un alto porcentaje de VPH-AR, factores de riesgo como $>3$ parejas sexuales y bajo uso de preservativo. Estos resultados resaltan la importancia de estrategias, no sólo, a nivel de la salud pública, sino también, de programas universitarios de educación, consejería y prevención a las jóvenes universitarias y a sus parejas, previo ingreso al programa de tamizaje para CCU. Finalmente, sería interesante, estudiar en cinco años más una población de adolescentes y jóvenes universitarias chilenas que hubieran recibido la vacuna para el VPH.

Agradecimientos: A todas las estudiantes universitarias que hicieron posible este estudio.

\section{Resumen}

Introducción: El inicio precoz de actividad sexual puede favorecer el desarrollo de alteraciones cervicales y de infecciones de transmisión sexual, en especial del virus papiloma humano (VPH) muy frecuente en adolescentes y jóvenes. Objetivo: Analizar el estado del cuello uterino, presencia del VPH y conductas sexuales en mujeres menores de 25 años. Material y Métodos: Participaron 182 estudiantes universitarias de 18-24 años, sanas, sexualmente activas y no vacunadas para VPH. Se realizó Papanicolaou (Pap) y clasificación del VPH en alto y bajo riesgo (AR y BR) mediante reacción de polimerasa en cadena en tiempo real. Las conductas sexuales fueron consultadas privadamente. Resultados: El 46,9\% de los Pap presentaron alteraciones citológicas (inflamación inespecífica/hemorrágico: $29,4 \%$ y frotis atípicos (FA):10,2\%). La frecuencia de los VPH-AR fue 24,3\%; de éstos, $67,4 \%$ presentó un Pap alterado. Hubo asociación entre alteraciones citológicas y presencia de VPH $(p<0,0001)$ y años de actividad sexual y FA o neoplasia intraepitelial grado I (NIE I) $(p=0,009)$. El $11,9 \%$ de las jóvenes estudiadas (21/177) presentó FA o NIE I, con 66,7\% de casos VPH-AR. Conclusiones: Estos hallazgos alertan la vulnerabilidad de estas jóvenes que tendrían un riesgo potencial de persistencia viral, NIE y eventualmente cáncer. Es importante enfatizar consejería y prevención previo a la edad normada de ingreso al programa de cribado para cáncer cérvico uterino en Chile.

\section{Referencias bibliográficas}

1.- MINSAL. Guías Clínicas AUGE, Cáncer Cérvico Uterino 2015;2da actualización. https:// www.minsal.cl/wp-content/uploads/2015/09/ GPC-CaCU.pdf.

2.- Hwang L Y, Ma Y, Benningfield S M, Clayton L, Hanson E N, Jay J, et al. Factors that influence the rate of epithelial maturation in the cervix in healthy young women. J Adolesc Health. 2009; 44 (2): 103-10. doi: 10.1016/j. jadohealth.2008.10.006.

3.- Satterwhite C L, Torrone E, Meites E, Dunne E F, Mahajan R, Ocfemia M C, et al. Sexually transmitted infections among US women and men: prevalence and incidence estimates, 2008. Sex Transm Dis. 2013; 40 (3): 187-93. doi: 10.1097/OLQ.0b013e318286bb53.

4.- Munoz N, Castellsague X, de González A B, Gissmann L. Chapter 1: HPV in the etiology of human cancer. Vaccine 2006; 24 Suppl 3: S3/1-10. Epub 2006/09/05. doi: 10.1016/j. vaccine.2006.05.115.

5.- Abraham A G, D’Souza G, Jing Y, Gange S J, Sterling T R, Silverberg M J, et al. Invasive cervical cancer risk among HIV-infected women: a North American multicohort collaboration prospective study. J Acquir Immune Defic Syndr. 2013; 62 (4): 405-13. doi: 10.1097/QAI.0b013e31828177d7.
6.- Ghosh I, Mandal R, Kundu P, Biswas J. Association of genital infections other than human papillomavirus with pre-invasive and invasive cervical neoplasia. J Clin Diagn Res. 2016; 10 (2): XE01-XE6. doi: 10.7860/ JCDR/2016/15305.7173.

7.- Ghosh I, Muwonge R, Mittal S, Banerjee D, Kundu P, Mandal R, et al. Association between high risk human papillomavirus infection and co-infection with Candida spp. and Trichomonas vaginalis in women with cervical premalignant and malignant lesions. J Clin Virol 2017; 87: 43-8. doi: 10.1016/j. jev.2016.12.007.

8.- Nonato DR, Alves RR, Ribeiro AA, Saddi VA, Segati KD, Almeida KP, et al. Prevalence and factors associated with coinfection of human papillomavirus and Chlamydia trachomatis in adolescents and young women. Am J Obstet Gynecol 2016; 215 (6): 753 e1- 753 e9. doi: 10.1016/j. ajog.2016.07.003.

9.- Melo A, Vásquez AM, Andana A, Matamala M, Pino T, Guzmán P, et al. Genotyping of human papillomavirus in women under 25 years old treated in the screening program for cervical cancer. Rev Chilena Infectol. 2014; 31 (5): 542 8. doi: 10.4067/S0716-10182014000500005.

10.- Dighe S B, Ajit D, Pathuthara S, Chinoy R. Papanicolaou stain: Is it economical to switch to rapid, economical, acetic acid, papanicolaou stain? Acta Cytol. 2006; 50 (6): 643-6. doi: 10.1159/000326034.

11.- Meneses E M M, Mota A, Viera E, Casimiro A, de Melo Cea M. HPV detection using primers MY09/MY11 and GP5+/GP6+ in patients with cytologic and/or colposcopic changes. J Bras Patol Med Lab 2014; 50 (4): 280-5. http:// dx.doi.org/10.5935/1676-2444.20140028.

12.- Noda T, Sasagawa T, Dong Y, Fuse H, Namiki M, Inoue M. Detection of human papillomavirus (HPV) DNA in archival specimens of benign prostatic hyperplasia and prostatic cancer using a highly sensitive nested PCR method. Urol Res. 1998; 26 (3): 165-9. PMID: 9694597.

13.- Fujinaga $Y$, Shimada M, Okazawa K, Fukushima M, Kato I, Fujinaga K. Simultaneous detection and typing of genital human papillomavirus DNA using the polymerase chain reaction. J Gen Virol. 1991; 72 ( Pt 5): 1039-44. doi: 10.1099/0022-131772-5-1039.

14.- Prado J C, Calleja-Macias I E, Bernard H U, Kalantari M, Macay S A, Allan B, et al. Worldwide genomic diversity of the human papillomaviruses-53, 56, and 66, a group of high-risk HPVs unrelated to HPV-16 and HPV-18. Virology 2005; 340 (1): 95-104. doi: 10.1016/j.virol.2005.06.024. 
15.- Kroupis C, Markou A, Vourlidis N, DionyssiouAsteriou A, Lianidou E S. Presence of high-risk human papillomavirus sequences in breast cancer tissues and association with histopathological characteristics. Clin Biochem 2006; 39 (7): 727-31. doi: 10.1016/j. clinbiochem.2006.03.005.

16.- Hernández-Arteaga S, López-Revilla R. Ultrasensitive quantitation of human papillomavirus type 16 E6 oncogene sequences by nested real time PCR. Infect Agents Cancer. 2010; 5: 9. doi: 10.1186/1750-9378-5-9.

17.- Agüero A C K, González-Blanco M. Neoplasia intraepitelial cervical de alto grado en mujeres menores de 25 años y mayores de 45 años. Rev Obstet Ginecol Venez 2012; 72 (2). http:// ve.scielo.org/pdf/og/v72n2/art04.pdf.

18.- Salas M N M, Petrosino P, Arenas A. Neoplasias intraepiteliales cervicales en adolescentes. Rev Obstet Ginecol Venez 2014; 74 (1). http://ve.scielo.org/pdf/og/v74n1/art05. pdf.

19.- Munro A, Powell R G, P AC, Bowen S, Spilsbury K, O'Leary P, et al. Spontaneous regression of CIN2 in women aged 18-24 years: a retrospective study of a state-wide population in Western Australia. Acta Obstet Gynecol Scand 2016; 95 (3): 291-8. doi: 10.1111/ aogs. 12835 .

20.- Baka S, Tsirmpa I, Chasiakou A, Tsouma I, Politi E, Gennimata V, et al. Inflammation on the cervical papanicolaou smear: evidence for infection in asymptomatic women? Infect Dis Obstet Gynecol 2013; 2013: 184302. doi: 10.1155/2013/184302.

21.- Bhutia K, Puri M, Gami N, Aggarwal K, Trivedi S S. Persistent inflammation on Pap smear: does it warrant evaluation? Indian J
Cancer 2011; 48 (2): 220-2. doi: 10.4103/0019$509 X .82901$.

22.- Brotman R M, Shardell M D, Gajer P, Tracy J K, Zenilman J M, Ravel J, et al. Interplay between the temporal dynamics of the vaginal microbiota and human papillomavirus detection. J Infect Dis. 2014; 210 (11): 172333. doi: 10.1093/infdis/jiu330.

23.- Mitra A, MacIntyre D A, Lee Y S, Smith A, Marchesi J R, Lehne B, et al. Cervical intraepithelial neoplasia disease progression is associated with increased vaginal microbiome diversity. Sci Rep. 2015; 5:16865. doi: 10.1038/ srep 16865

24.- Dareng E O, Ma B, Famooto A O, Adebamowo S N, Offiong R A, Olaniyan O, et al. Prevalent high-risk HPV infection and vaginal microbiota in Nigerian women. Epidemiol Infect 2016; 144 (1): 123-37. doi: 10.1017/S0950268815000965.

25.- Gao W, Weng J, Gao Y, Chen X. Comparison of the vaginal microbiota diversity of women with and without human papillomavirus infection: a cross-sectional study. BMC Infectious Dis 2013; 13: 271. doi: 10.1186/1471-2334-13-271.

26.- Almonte M, Murillo R, Sanchez GI, Jeronimo J, Salmeron J, Ferreccio C, et al. New paradigms and challenges in cervical cancer prevention and control in Latin America. Salud Publica Mex 2010; 52 (6): 544-59. PMID: 21271014

27.- Roset Bahmanyar E, Paavonen J, Naud P, Salmeron J, Chow SN, Apter D, et al. Prevalence and risk factors for cervical HPV infection and abnormalities in young adult women at enrolment in the multinational PATRICIA trial. Gynecol Oncol 2012; 127 (3): 440-50. doi: 10.1016/j.ygyno.2012.08.033.

28.- Villegas-Castaño A T-A, L. Prevalencia de infecciones de transmisión sexual y factores de riesgo para la salud sexual de adolescentes escolarizados, Medellín, Colombia, 2013. Iatreia 2016; 29 (1): 5-17. http://dx.doi. org/10.17533/udea.iatreia.v29n1a11.

29.- Castellsague $X$, Paavonen J, Jaisamrarn U, Wheeler C M, Skinner S R, Lehtinen M, et al. Risk of first cervical HPV infection and precancerous lesions after onset of sexual activity: analysis of women in the control arm of the randomized, controlled PATRICIA trial. BMC Infect Dis. 2014; 14: 551. doi: 10.1186/s12879014-0551-y.

30.- Rodríguez G C C, Alonso R, Sica A, Segredo S, León I, Musé I. Conocimientos, actitudes y prácticas sobre el test de Papanicolaou y estadificación del cáncer de cuello uterino. Rev Méd Urug 2015; 31 (4): 231-40. http://www.scielo.edu.uy/pdf/rmu/ v31n4/v31n4a02.pdf

31.- Villegas N, Cianelli R, Santisteban D, Lara L, Vargas J. Factores que influencian la adquisicion de infecciones de transmision sexual y VIH en mujeres jovenes chilenas que participaron en la intervencion online I-STIPI. Hisp Health Care Int 2016; 14 (1): 47-56. doi: $10.1177 / 1540415316629682$.

32.- Eguiluz-Cárdenas I, Torres-Pereda P, AllenLeigh B. Percepciones sobre uso de condón e ITS/VIH: migrantes y no-migrantes de México a EE. UU. Revista Latinoamericana de Ciencias Sociales Niñez y Juventud 2013; 11 (2): 51526. http://www.scielo.org.co/pdf/rlcs/v11n2/ v11n2a05.pdf.

33.- Burd E M. Human papillomavirus laboratory testing: the changing paradigm. Clin Microbiol Rev. 2016; 29 (2): 291-319. doi: 10.1128/ CMR.00013-15. 